\title{
A low incidence of perineal hernia when using a biological mesh after extralevator abdominoperineal excision with or without pelvic exenteration or distal sacral resection in locally advanced rectal cancer patients
}

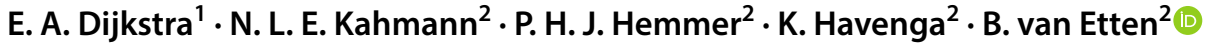

Received: 19 March 2020 / Accepted: 19 May 2020 / Published online: 8 June 2020

(c) The Author(s) 2020

\begin{abstract}
Background Extralevator abdominoperineal excision (ELAPE), abdominoperineal excision (APE) or pelvic exenteration (PE) with or without sacral resection (SR) for locally advanced rectal cancer leaves a significant defect in the pelvic floor. At first, this defect was closed primarily. To prevent perineal hernias, the use of a biological mesh to restore the pelvic floor has been increasing. The aim of this study, was to evaluate the outcome of the use of a biological mesh after ELAPE, APE or PE with/without SR.

Methods A retrospective study was conducted on patients who had ELAPE, APE or PE with/without SR with a biological mesh (Permacol ${ }^{\mathrm{TM}}$ ) for pelvic reconstruction in rectal cancer in our center between January 2012 and April 2015. The endpoints were the incidence of perineal herniation and wound healing complications.

Results Data of 35 consecutive patients [22 men, 13 women; mean age 62 years (range 31-77 years)] were reviewed. Median follow-up was 24 months (range 0.4-64 months). Perineal hernia was reported in 3 patients (8.6\%), and was asymptomatic in 2 of them. The perineal wound healed within 3 months in $37.1 \%(n=13)$, within 6 months in $51.4 \%(n=18)$ and within 1 year in $62.9 \%(n=22)$. In $17.1 \%(n=6)$, the wound healed after 1 year. It was not possible to confirm perineal wound healing in the remaining 7 patients $(20.0 \%)$ due to death or loss to follow-up. Wound dehiscence was reported in 18 patients (51.4\%), 9 of whom needed vacuum-assisted closure therapy, surgical closure or a flap reconstruction.

Conclusions Closure of the perineal wound after (EL)APE with a biological mesh is associated with a low incidence of perineal hernia. Wound healing complications in this high-risk group of patients are comparable to those reported in the literature.
\end{abstract}

Keywords Biological mesh $\cdot$ Permacol $\cdot$ Perineal hernia $\cdot$ Wound healing $\cdot$ Rectal cancer surgery $\cdot$ Extralevator abdomino perineal excision $\cdot$ Pelvic exenteration

B. van Etten

b.van.etten@umcg.nl

1 Department of Medical Oncology, University of Groningen, University Medical Center Groningen, Groningen, The Netherlands

2 Department of Surgery, University of Groningen, University Medical Center Groningen, Hanzeplein 1, P.O. Box 30.001, 9700 RB Groningen, The Netherlands

\section{Introduction}

Abdominoperineal excision (APE) is widely used in the treatment of rectal cancer when the tumour is less than $6 \mathrm{~cm}$ from the anal verge. The conventional technique of APE is an abdominal mobilization of the mesorectum to the distal rectum/anus followed by perineal excision close to the anal sphincter. During an extralevator APE (ELAPE), the levator ani muscle is resected en bloc with the rectum [1]. Because of this, the defect in the pelvic floor after ELAPE is larger than after conventional APE. There is a comparable perineal wound morbidity between ELAPE and conventional APE though $[2,3]$. In ventrally advanced rectal tumours pelvic 
exenteration (PE) or resection of the posterior vaginal wall, and in dorsally advanced tumours, distal sacral resection (SR) may be considered in combination with (EL)APE. In these types of resection, the defect in the pelvic floor is more prominent, and filling of the pelvis is mainly by omentoplasty or small bowel. This significant defect in the pelvic floor may result in lower rates of wound healing.

Primary closure of the perineal wound is associated with a high rate of wound complications, especially in patients treated with neoadjuvant radiotherapy (40-45\%) [4-7]. Other options for closure are a myocutaneous flap reconstruction, a lotus petal flap reconstruction or a biological mesh. A myocutaneous flap reconstruction and biological mesh reconstruction are associated with equal morbidity [8]. However, gluteal flap reconstructions result in a higher perineal hernia rate than those with biological mesh $(21 \%$ vs $0, p<0.01$ ) [9]. After a lotus petal flap reconstruction, Clavien-Dindo grade I-II complications occurred in $46 \%$ of patients [10]. The use of a biological mesh is promising since the perineal herniation rate has been lowered to $0-13 \%$ $[4,9,11-14]$. Besides, a systematic review showed improved pelvic wound healing, shortened operation time and earlier postoperative mobilization after biological mesh compared to primary closure [15].

In the University Medical Center Groningen (UMCG), we have used biological mesh reconstructions after ELAPE, APE with or without PE or SR between January 2012 and April 2015. In this paper, we describe our results with an emphasis on perineal hernia and wound healing.

\section{Materials and methods}

A total of consecutive 35 patients who had surgery for rectal cancer with or without PE or SR with the reconstruction of the pelvic floor using a biological mesh (Permacol ${ }^{\mathrm{TM}}$; Covidien, Mansfield, MA, USA), at the UMCG between January 2012 and April 2015, were evaluated. Patient, preoperative, surgery and complication and toxicity characteristics were collected.

All patients had pelvic floor reconstruction with a Permacol $^{\mathrm{TM}}$ Surgical implant. This Permacol ${ }^{\mathrm{TM}}$ mesh is a porcine dermal collagen implant from which cells, ribonucleic acid and deoxyribonucleic acid are removed. The resulting acellular collagen matrix is then cross-linked to enhance durability. The size of the Permacol ${ }^{\mathrm{TM}}$ mesh depended on the size of the defect of the pelvic floor. The mesh was fixated with prolene sutures to the pelvic sidewall and the remnants of the levator muscle, the presacral fascia and the remnants of the puborectal sling. This part of the operation was performed in a prone jackknife or knee-chest position. All patients had reconstruction by omentoplasty. First, adhesions of the small bowel, colon or abdominal omentum were released. Then the omentum was cleared from the transverse colon and the stomach. The omentoplasty was pedicled on the right gastroepiploic artery and placed in the small pelvis. However, in some cases, the defect area was also covered with a de-epithelialized lotus petal flap. The flap was de-epithelialized so the extensive soft tissue could be used to fill dead spaces to prevent fluid accumulation causing complications. The lotus petal flap reconstruction technique is a perforator flap which can be mono- or bilateral and has been described earlier by our group [10]. In short, in the gluteal or inguinal fold the length and width of the flap are marked preoperatively. After resection, the skin of the flap is de-epithelialized and incised until the underlying fascia of the muscle is mobilized by elevating the flap from lateral to medial. The skin is closed in layers and finished with sutures.

Complications were graded using the Clavien-Dindo classification [15]. We did not differentiate between early and late complications. The treatment of wound complications was divided into five groups with conservative treatment, antibiotics, vacuum-assisted closure (VAC), surgical re-intervention or a secondary lotus petal flap reconstruction as possible treatment options. The following United States Food and Drug Administration (FDA) definition of complete wound healing was used: $100 \%$ re-epithelialization of the wound surface with no discernable exudate and without drainage or dressing requirement, confirmed at outpatient clinic visits [16]. A computed tomography (CT) scan was used in the follow-up of these rectal cancer patients and also to detect perineal hernia.

The association between wound dehiscence and multiple variables (sex, body mass index (BMI), smoking, diabetes mellitus, corticosteroids, cancer stage, American Society of Anesthesiologists (ASA) classification, neoadjuvant therapy, type of surgery, lotus petal flap reconstruction, intraoperative brachytherapy (IOBT), wound closure, perineal hernia, decease and cause of death) were calculated using the Pearson's Chi-square test. A $p$-value of $<0.05$ was considered significant. All analyses were performed using SPSS, version 23.0 (SPSS, Chicago, IL, USA).

\section{Results}

\section{Patient characteristics}

Table 1 shows the baseline characteristics. The median length of follow-up was 24 months (range 0.4-64 months). Almost all patients had resection for a primary or recurrent rectal adenocarcinoma; one patient was treated for a rectal gastrointestinal stromal tumour (GIST). All of the 
Table 1 Baseline characteristics

\begin{tabular}{|c|c|c|c|}
\hline & $\begin{array}{l}\text { Total patient group } \\
(n=35)\end{array}$ & $\begin{array}{l}\text { Wound dehiscence } \\
(n=18)\end{array}$ & $p$ value \\
\hline Sex & & & 0.90 \\
\hline Male & $22(62.9)$ & $11(61.1)$ & \\
\hline Female & $13(37.1)$ & 7 (38.9) & \\
\hline Age at randomization in years* & $62(31-77)$ & $60(31-75)$ & \\
\hline $\mathrm{BMI} \mathrm{kg} / \mathrm{m}^{2 * *}$ & $28(20-38)$ & $27(18-38)$ & \\
\hline Smoking & & & 0.56 \\
\hline Yes & $9(25.7)$ & $6(33.3)$ & \\
\hline No & $26(74.3)$ & $12(66.7)$ & \\
\hline Diabetes mellitus & & & 0.97 \\
\hline Yes & $4(11.4)$ & $2(11.1)$ & \\
\hline No & $31(88.6)$ & $16(88.9)$ & \\
\hline Corticosteroids & & & 0.34 \\
\hline Yes & $5(14.3)$ & $1(5.6)$ & \\
\hline No & $30(85.7)$ & $17(94.4)$ & \\
\hline Indication & & & 0.61 \\
\hline GIST & $1(2.9)$ & $0(0.0)$ & \\
\hline Adenocarcinoma & $33(97.1)$ & $18(100.0)$ & \\
\hline $\mathrm{T} 3$ & $8(22.9)$ & $4(22.2)$ & \\
\hline $\mathrm{T} 4$ & $16(45.7)$ & $6(33.3)$ & \\
\hline Recurrence & $10(28.6)$ & $8(44.4)$ & \\
\hline ASA physical status & & & 0.48 \\
\hline 1 & $8(23.5)$ & $3(16.7)$ & \\
\hline 2 & $24(70.6)$ & $15(83.3)$ & \\
\hline 3 & $2(5.7)$ & $0(0.0)$ & \\
\hline
\end{tabular}

$B M I$ body mass index, GIST gastrointestinal stromal tumour, ASA American Society of Anesthesiologists Data are presented as $N(\%)$.*mean (range), **median(range)

adenocarcinoma patients had neoadjuvant radiotherapy (Table 2).

\section{Treatment characteristics}

In total, 35 consecutive patients had surgery for rectal cancer and a Permacol ${ }^{\mathrm{TM}}$ mesh was used. Nine patients (25.7\%) had previous surgery for rectal cancer. In total, an ELAPE was performed in 13 patients (37.1\%) and an APE in 15 patients $(42.9 \%)$. Excision of the distal sacrum was performed in $61.5 \%$ of the ELAPE patients and in $66.7 \%$ of the APE patients. The other patients $(20.0 \%)$ underwent $\mathrm{PE}$, which includes excision of the uterus, vagina and/or bladder in female, or bladder and prostate in male patients (Table 2).

All patients had reconstruction by omentoplasty. In 7 patients $(20.0 \%)$, a lotus petal flap reconstruction was performed immediately following the rectal resection. In 2 other patients, a lotus petal flap reconstruction was performed secondarily, because of invalidating wound healing problems after initial primary skin closure.
Three patients $(8.6 \%)$ received intraoperative brachitherapy (IOBT) with a dose of 10 Gy during surgery, because of a peroperatively suspected close resection margin. In all 3 patients, there was postoperative wound dehiscence.

\section{Wound dehiscence}

Eighteen patients (51.4\%) had minor to major wound dehiscence, for which treatment was conservative in 8 cases (grade I complication). One patient received antibiotic treatment (grade II complication). A wound dehiscence grade III complication occurred in 9 patients $(27 \%)$ : 5 patients needed VAC therapy, and the other 4 patients required a reoperation with the use of lotus petal flap in 2 cases (Table 3).

None of the variables sex, BMI, smoking, diabetes mellitus, corticosteroids, cancer stage, ASA classification, neoadjuvant therapy, type of surgery, lotus petal flap reconstruction, IOBT wound closure, perineal hernia, 
Table 2 Treatment characteristics

\begin{tabular}{lccc}
\hline & $\begin{array}{c}\text { Total patient group } \\
(n=35)\end{array}$ & $\begin{array}{l}\text { Wound dehiscence } \\
(n=18)\end{array}$ & $p$ value \\
\hline Neoadjuvant radiotherapy & & & 0.67 \\
$50.0 / 50.4$ Gy & $20(57.1)$ & $9(61.1)$ & \\
$30.0 / 30.6$ Gy & $7(20.0)$ & $6(33.3)$ & \\
$5 \times 5$ Gy & $7(20.0)$ & $0(16.7)$ & 0.56 \\
No radiotherapy (GIST tumor) & $1(2.9)$ & $3(16.7)$ & \\
Surgery & & $1(5.6)$ & \\
ELAPE without distal sacrum resection & $8(22.9)$ & $4(22.2)$ & \\
ELAPE with distal sacrum resection & $5(14.3)$ & $8(44.4)$ & \\
APE without distal sacrum resection & $5(14.3)$ & $2(11.1)$ & \\
APE with distal sacrum resection & $10(28.6)$ & $5(27.8)$ & \\
Pelvic exenteration & $7(20.0)$ & $13(72.2)$ & \\
Lotus petal flap & & $3(20.0)$ & \\
Yes & $28(80.0)$ & $15(83.3)$ & \\
No & & $3(8.6)$ & \\
IOBT & $32(91.4)$ & \\
Yes & & \\
No & &
\end{tabular}

Data are presented as $N(\%)$

ELAPE extralevator abdominoperineal resection, APE abdominoperineal resection, GIST gastrointestinal stromal tumour, IOBT intraoperative brachytherapy
Table 3 Wound characteristics

\begin{tabular}{lcll}
\hline & $\begin{array}{l}\text { Total patient group } \\
(n=35)\end{array}$ & $\begin{array}{l}\text { Wound dehiscence } \\
(n=18)\end{array}$ & $p$ value \\
\hline $\begin{array}{l}\text { Wound healing } \\
\text { 0-30 days }\end{array}$ & $2(5.7)$ & $0(0.0)$ & 0.79 \\
30-90 days & $11(31.4)$ & $3(16.7)$ & \\
90-180 days & $5(14.3)$ & $3(16.7)$ & \\
180-365 days & $4(11.4)$ & $3(16.7)$ & \\
$>365$ days & $6(17.1)$ & $4(22.2)$ & \\
Deceased* & $4(11.4)$ & $2(11.1)$ & \\
Lost to follow-up & $3(8.6)$ & $3(16.7)$ & \\
Wound dehiscence & & $8(23.5)$ & \\
Clavien-Dindo I & & $9(5.6)$ & \\
Clavien-Dindo II & & $9(50.0)$ & \\
Clavien-Dindo & & & \\
III & & &
\end{tabular}

Data are presented as $N(\%)$

*Unclear whether the wound was healed before death

decease and cause of death were associated with an increased risk of wound dehiscence (Tables 1, 2, 3, 4).

\section{Wound healing}

Table 3 shows wound healing (according to the FDA definition of a completely healed wound [16]) and wound complications following surgery with Permacol $^{\mathrm{TM}}$ mesh closure. The wound was healed within 30 days after surgery in 2 patients $(5.7 \%)$ and within 3 months after surgery in 11 patients $(31.4 \%)$. In total, the wound was completely healed within 1 year after surgery in 22 patients $(62.9 \%)$. As regards the remaining 13 patients $(37.1 \%)$, the wound was completely healed after 1 year in 6 patients $(17.1 \%), 3$ patients (8.6\%) were lost to follow-up and 4 patients (11.4\%) died before confirmation of wound healing during outpatient clinical visits (Table 3).

One patient experienced a deep wound infection 3 weeks after the initial surgery. The Permacol ${ }^{\mathrm{TM}}$ mesh lay loose in the pelvis and it was removed.

\section{Perineal hernia}

A perineal hernia occurred in 3 patients $(8.6 \%)$. This happened 10, 13 and 23 months after surgery. None of them experienced a wound infection after surgery and before the occurrence of the perineal hernia. One of the patients with perineal hernia smoked and had diabetes mellitus, another patient smoked, and the third patient used corticosteroids. Two of the hernias were asymptomatic, so no further treatment was necessary. The other patient underwent surgical correction of the hernia (Table 4). Two of the patients with an asymptomatic perineal hernia had an ELAPE, the other patient ( with a symptomatic perineal hernia) an APE. Of 
Table 4 Outcome

\begin{tabular}{llll}
\hline & $\begin{array}{l}\text { Total patient group } \\
(n=35)\end{array}$ & $\begin{array}{l}\text { Wound dehiscence } \\
(n=18)\end{array}$ & $p$ value \\
\hline Perineal hernia & & 0.20 \\
$\quad$ Cases & $3(8.6)$ & $0(0.0)$ & \\
$\quad \begin{array}{l}\text { Asymptomatic } \\
\text { Symptomatic }\end{array}$ & $2(66.6)$ & $0(0.0)$ & 0.50 \\
Deceased & $1(33.3)$ & $11(61.1)$ & \\
Cases & $18(51.4)$ & $25(2-47)$ & 0.83 \\
$\quad$ Months after surgery* & $24(0.3-67)$ & $8(72.7)$ & \\
Cause of death & $14(77.8)$ & $1(9.1)$ & \\
$\quad$ Tumour recurrence and/or & & $0(0.0)$ & \\
$\quad$ metastases & $1(5.6)$ & $2(18.2)$ & \\
Sepsis and MOF & $1(5.6)$ & & \\
Pulmonary embolism & $2(11.1)$ & & \\
Unknown & &
\end{tabular}

MOF multi-organ failure

Data are presented as $N(\%) *$ mean(range)

the 7 patients who were lost to follow-up or died before the wound was healed, only 1 patient $(14.3 \%)$ developed a perineal hernia.

\section{Mortality}

In total, 18 patients $(51.4 \%)$ died, one of them within 30 days after surgery, due to a pulmonary embolism on day 11. Another patient died as a result of sepsis and multiorgan failure within 3 months after surgery. In 11 patients progression and/or recurrence of disease was the cause of death during follow-up. The cause of death was unknown in 3 patients (Table 4 ).

\section{Discussion}

In this study, we demonstrated a low rate of perineal hernia after the use of a biological mesh in rectal cancer patients. Moreover half of the perineal wounds closed within 6 months.

The perineal hernia rate when a biological mesh was used was $8.6 \%$. Only 2 patients $(5.7 \%)$ experienced a perineal hernia after ELAPE, which were both asymptomatic. The currently most extensive series of biological mesh repair after ELAPE, reported by Thomas et al., demonstrated a comparable asymptomatic perineal hernia rate of 7\% [17]. When biological mesh was combined with a lotus petal flap, the incidence of perineal hernia was low $(n=1)$ in our study. This perineal hernia rate is comparable to that in the study of Hellinga et al. in which none of the patients experienced a perineal hernia after the combination of a lotus petal flap with a biological mesh [10]. In the multicenter
BIOPEX-study, 101 patients were randomized between primary closure of the perineum or closure with a biological mesh (non-crosslinked Strattice ${ }^{\mathrm{TM}}$ Reconstructive Tissue Matrix from LifeCell, Allergan ${ }^{\odot}$ ) [13]. After 1-year of follow-up, the perineal hernia rate was significantly lower in patients with a biological mesh (13\% after biological mesh, $27 \%$ after primary closure, $p=0.03$ ) [13]. Besides, among the 228 patients in a retrospective study, significantly less perineal hernias developed after use of a biological mesh instead of primary closure (3.4\% vs $13.0 \%, p=0.02)$ [11]. In the study of Sayers et al., 56 pateints underwent ELAPE had primary closure with $(n=8)$ or without $(n=38)$ biologic mesh or flap [18]. A total of 14 patients (25.9\%) developed a perineal hernia including both patients who received a biological mesh reconstruction, and 12 of the 38 patients who were closed with sutures [18]. The retrospective study of Christensen et al. compared the use of a gluteal flap with a biological mesh in 57 patients [9]. A perineal hernia developed in 7 patients with a gluteal flap reconstruction vs. none of the patients in the mesh group $(p<0.01)$ [9]. It seems that the chance of developing a perineal hernia is smaller after using a biological mesh than with primary closure and gluteal flap. However, data is limited. The clinical review by Foster et al. stated that the decision regarding which method should be performed should depend on surgical expertise, morbidity, cost-effectiveness and the size of the defect [7].

More than half of the patients in our study experienced minor to major wound healing problems. Half of them $(n=9)$ needed invasive treatment for these problems. One patient experienced a deep wound infection 3 weeks after the initial surgery. The mesh lay loose in the pelvis and it has been removed. Just like in our study, all patients in the BIOPEX-study received preoperative radiotherapy 
[13]. The BIOPEX-study showed no significant difference in perineal wound complications 30 days after surgery between the primary closure $(66 \%)$ and biological mesh $(63 \%)$ groups $(p=0.72)$ [13]. In the prospective study of Peacock et al., all 34 patients received a biological mesh, and $82,4 \%$ received radiotherapy up to 45 Gy [12]. This study reports an overall rate of perineal wound complications of $32 \%, 8 \%$ of which needed invasive treatment [12]. Grade III wound complication occurred in 7 patients (7\%) in the study by Thomas et al 4 of whom needed VAC therapy [17]. In total, $71 \%$ of patients in this observational study were preoperatively irradiated with 45 Gy [17]. Christensen et al. reported a higher perineal wound infection rate after the use of biological mesh (17\%) compared to a gluteal flap reconstruction $(6 \%)$ although this difference was found not significant $(p=0.26)$ [9]. However, Christensen et al. described wound infection as an infection requiring surgical intervention by operative irrigation and/or debridement or VAC [9]. If we use the same criteria, we find a wound infection rate of $14 \%$. In total, $44 \%$ of patients in the study of Sayers et al., experienced perineal complications. Twenty-two of them (39.3\%) received preoperative radiotherapy [18]. On the other hand, none of the patients $(n=17)$ in the study by Ge et al., developed perineal complications after the use of a biological mesh [19]. However, the patients in this study did not receive preoperative radiotherapy [19]. In preoperatively irradiated patients who had APE with primary closure, wound complications occur in 40-45\% [4-6].

In an ongoing multicenter trial, the NEAPE-trial, 487 patients will be randomized (1:1) between the use of a porcine-collagen implant or the use of a gluteus maximus muscle. The aim is to investigate whether a porcine-collagen implant is superior or equally beneficial to a gluteus maximus reconstruction. We are awaiting the results.

Wound healing problems in this specific surgery, are most likely caused by multiple factors. The most critical risk factor, which also emerges from the literature above, is preoperative radiotherapy $[4-6,11,20]$. A long operative time, more extensive surgery, contamination of the operating field, conventional primary closure, intraoperative bowel-perforation and the location of the wound are other possible risk factors $[6,11,21]$. With the use of a biological mesh, an anatomical dead space is created between the mesh and perineal skin, in which exudate can lead to abscess formation.

Limitations of our study include the small study population and the retrospective nature of the study. Another limitation is that the use of the biological mesh was not compared with other methods of wound closure. There was no systematic follow-up interval regarding wound inspection and no scale for wound observation was used.

\section{Conclusions}

Our study demonstrates that the use of a biological mesh is a safe and feasible method for pelvic reconstruction and wound closure after ELAPE, APE or PE with or without SR in patients with locally advanced or recurrent rectal cancer and multiple wound healing risk factors. Moreover, the use of biological mesh is associated with a low rate of perineal hernia.

Authors' contribution statement All authors contributed to the study conception and design. The first draft of the manuscript was written by Esmée Anne Dijkstra and all authors commented on previous versions of the manuscript. All authors read and approved the final manuscript.

\section{Compliance with ethical standards}

Funding No grant support or financial relationship to declare by any of the authors.

Conflict of interest The authors declare that they have no conflict of interest.

Ethical approval Ethical approval was waived by the local Ethics Committee of University Medical Center Groningen in view of the retrospective nature of the study and all the procedures being performed were part of the routine care.

Consent to participate No participants information and consent statement apply to these retrospective research activities. This was approved by the local Ethics Committee of University Medical Center Groningen. The reasons for this are that Permacol mesh are used more often and are therefore part of standard of care, and because some of the patients will be dead at the end of the study (high mortality rate of rectal cancer). As a result, the researchers cannot reasonably be expected to check all this data for informed consent. In addition, this research is in public interest. Furthermore, this research cannot be conducted without this data. Also, the patients included in the study may not appear in the 'Geen bezwaar register'. We rely on Article 458 of the WGBO.

Consent to publish Not applicable.

Data and/or Code availability The datasets during and/or analysed during the current study are available from the corresponding author on reasonable request.

Informed consent For this type of study formal consent is not required.

Open Access This article is licensed under a Creative Commons Attribution 4.0 International License, which permits use, sharing, adaptation, distribution and reproduction in any medium or format, as long as you give appropriate credit to the original author(s) and the source, provide a link to the Creative Commons licence, and indicate if changes were made. The images or other third party material in this article are included in the article's Creative Commons licence, unless indicated otherwise in a credit line to the material. If material is not included in the article's Creative Commons licence and your intended use is not permitted by statutory regulation or exceeds the permitted use, you will need to obtain permission directly from the copyright holder. To view a copy of this licence, visit http://creativecommons.org/licenses/by/4.0/. 


\section{References}

1. Holm T, Ljung A, Häggmark T et al (2007) Extended abdominoperineal resection with gluteus maximus flap reconstruction of the pelvic floor for rectal cancer. Br J Surg 94:232-238. https:// doi.org/10.1002/bjs.5489

2. Yang Y, Xu H, Shang Z et al (2015) Outcome of extralevator abdominoperineal excision over conventional abdominoperineal excision for low rectal tumor: a meta-analysis. Int J Clin Exp Med 8:14855-14862

3. Zhou X, Sun T, Xie H et al (2015) Extralevator abdominoperineal excision for low rectal cancer: a systematic review and meta-analysis of the short-term outcome. Color Dis 17:474-481. https://doi. org/10.1111/codi.12921

4. Bullard KM, Trudel JL, Baxter NN, Rothenberger DA (2005) Primary perineal wound closure after preoperative radiotherapy and abdominoperineal resection has a high incidence of wound failure. Dis Colon Rectum 48:438-443. https://doi.org/10.1007/ s10350-004-0827-1

5. Chadwick MA, Vieten D, Pettitt E et al (2006) Short course preoperative radiotherapy is the single most important risk factor for perineal wound complications after abdominoperineal excision of the rectum. Color Dis 8:756-761. https://doi.org/10.111 1/j.1463-1318.2006.01029.x

6. Artioukh DY, Smith RA, Gokul K (2007) Risk factors for impaired healing of the perineal wound after abdominoperineal resection of rectum for carcinoma. Color Dis 9:362-367. https:// doi.org/10.1111/j.1463-1318.2006.01159.x

7. Foster JD, Tou S, Curtis NJ et al (2018) Closure of the perineal defect after abdominoperineal excision for rectal adenocarcinoma-ACPGBI position statement. Color Dis 20:5-23. https:// doi.org/10.1111/codi.14348

8. Peacock O, Pandya H, Sharp T et al (2012) Biological mesh reconstruction of perineal wounds following enhanced abdominoperineal excision of rectum (APER). Int J Colorectal Dis 27:475-482. https://doi.org/10.1007/s00384-011-1325-2

9. Christensen HK, Nerstrøm P, Tei T, Laurberg S (2011) Perineal repair after extralevator abdominoperineal excision for low rectal cancer. Dis Colon Rectum 54:711-717. https://doi.org/10.1007/ DCR.0b013e3182163c89

10. Hellinga J, Khoe PCKH, van Etten B et al (2016) Fasciocutaneous lotus petal flap for perineal wound reconstruction after extralevator abdominoperineal excision: application for reconstruction of the pelvic floor and creation of a neovagina. Ann Surg Oncol 23:4073-4079. https://doi.org/10.1245/s10434-016-5332-y

11. Han JG, Wang ZJ, Gao ZG et al (2019) Perineal wound complications after extralevator abdominoperineal excision for low rectal cancer. Dis Colon Rectum 62:1477-1484. https://doi.org/10.1097/ DCR.0000000000001495

12. Peacock O, Simpson JA, Tou SI et al (2014) Outcomes after biological mesh reconstruction of the pelvic floor following extralevator abdominoperineal excision of rectum (APER). Tech Coloproctol 18:571-577. https://doi.org/10.1007/s10151-013-1107-7

13. Musters GD, Klaver CEL, Bosker RJI et al (2017) Biological mesh closure of the pelvic floor after extralevator abdominoperineal resection for rectal cancer: a multicenter randomized controlled trial (the BIOPEX-study). Ann Surg 265:1074-1081. https://doi. org/10.1097/SLA.0000000000002020

14. Wille-Jørgensen P, Pilsgaard B, Møller P (2009) Reconstruction of the pelvic floor with a biological mesh after abdominoperineal excision for rectal cancer. Int J Colorectal Dis 24:323-325. https ://doi.org/10.1007/s00384-008-0607-9

15. Schiltz B, Buchs NC, Penna M et al (2017) Biological mesh reconstruction of the pelvic floor following abdominoperineal excision for cancer: a review. World J Clin Oncol 8:249-254. https://doi. org/10.5306/wjco.v8.i3.249

16. Gould L, Li WW (2019) Defining complete wound closure: closing the gap in clinical trials and practice. Wound Repair Regen. https://doi.org/10.1111/wrr.12707

17. Thomas PW, Blackwell JEM, Herrod PJJ et al (2019) Longterm outcomes of biological mesh repair following extra levator abdominoperineal excision of the rectum: an observational study of 100 patients. Tech Coloproctol 23:761-767. https://doi. org/10.1007/s10151-019-02056-0

18. Sayers AE, Patel RK, Hunter IA (2015) Perineal hernia formation following extralevator abdominoperineal excision. Color Dis 17:351-355. https://doi.org/10.1111/codi.12843

19. Ge W, Jiang S, Qi W et al (2017) Extralevator abdominoperineal excision for rectal cancer with biological mesh for pelvic floor reconstruction. Oncotarget 8:8818-8824. https://doi.org/10.18632 /oncotarget. 12502

20. Musters GD, Buskens CJ, Bemelman WA, Tanis PJ (2014) Perineal wound healing after abdominoperineal resection for rectal cancer: a systematic review and meta-analysis. Dis Colon Rectum 57:1129-1139. https://doi.org/10.1097/DCR.0000000000000182

21. Christian CK, Kwaan MR, Betensky RA et al (2005) Risk factors for perineal wound complications following abdominoperineal resection. Dis Colon Rectum 48:43-48. https://doi.org/10.1007/ s10350-004-0855-x

Publisher's Note Springer Nature remains neutral with regard to jurisdictional claims in published maps and institutional affiliations. 